\title{
Review
}

\section{Will Remotely Based Pulmonary Rehabilitation Water Down Its Effectiveness?}

\author{
Himanshu Rawal ${ }^{1}$, Sharon D. Cornelison ${ }^{2}{ }^{\circledR}$, Sheryl M. Flynn ${ }^{3}$ and Jill A. Ohar ${ }^{1, *}$ \\ 1 Department of Internal Medicine, Section on Pulmonary Medicine, Critical Care, Allergy and Immunologic \\ Diseases, Atrium Health Wake Forest Baptist, Medical Center Boulevard, Winston-Salem, NC 27157, USA; \\ hrawal@wakehealth.edu \\ 2 Department of Pulmonary and Cardiac Rehabilitation, J. Paul Sticht Center on Aging and Rehabilitation, \\ Atrium Health Wake Forest Baptist, Medical Center Boulevard, Winston-Salem, NC 27157, USA; \\ scorneli@wakehealth.edu \\ 3 Blue Marble Health, Lincoln Avenue, Altadena, CA 91001, USA; sheryl@bluemarblehealthco.com \\ * Correspondence: johar@wakehealth.edu
}

Citation: Rawal, H.; Cornelison, S.D Flynn, S.M.; Ohar, J.A. Will Remotely Based Pulmonary Rehabilitation Water Down Its Effectiveness? Life 2021, 11, 1270. https://doi.org/ $10.3390 /$ life11111270

Academic Editors: Claudio F. Donner, Linda Nici and Richard Zuwallack

Received: 28 October 2021

Accepted: 17 November 2021

Published: 20 November 2021

Publisher's Note: MDPI stays neutral with regard to jurisdictional claims in published maps and institutional affiliations.

Copyright: (c) 2021 by the authors. Licensee MDPI, Basel, Switzerland. This article is an open access article distributed under the terms and conditions of the Creative Commons Attribution (CC BY) license (https:// creativecommons.org/licenses/by/ $4.0 /)$.

\begin{abstract}
Despite numerous benefits, traditional Pulmonary Rehabilitation (PR) as a resource remains underutilized in chronic lung disease. Less than $3 \%$ of eligible candidates for PR attend one or more sessions after hospitalization due to many barriers, including the ongoing COVID-19 pandemic. Emerging alternative models of PR delivery such as home-based PR, tele-rehabilitation, web-based PR, or hybrid models could help address these barriers. Numerous studies have tested the feasibility, safety, and efficacy of these methods, but there is wide variability across studies and methods. We conducted a literature review to help determine if these alternative delivery methods watered down the effectiveness of PR. To evaluate the effectiveness of remotely based PR, the authors performed a literature search for randomized controlled trials (RCTs), cohort studies, and case series using PubMed, CINAHL, and Medline to identify relevant articles through 1 May 2021. Twenty-six applicable studies were found in which 11 compared tele-rehabilitation to conventional clinic-based PR; 11 evaluated tele-rehabilitation using the patient's baseline status as control; and four compared tele-rehabilitation to no rehabilitation. Despite the different technologies used across studies, telerehabilitation was found to be both a feasible and an efficacious option for select patients with lung disease. Outcomes across these studies demonstrated similar benefits to traditional PR programs. Thus the existing data does not show that remotely based PR waters down the effectiveness of conventional PR. Use of remotely based PR is a feasible and effective option to deliver PR, especially for patients with significant barriers to conventional clinic-based PR. Additional, well-conducted RCTs are needed to answer the questions regarding its efficacy, safety, cost-effectiveness and who, among patients with COPD and other lung diseases, will derive the maximum benefit.
\end{abstract}

Keywords: remotely; tele-rehabilitation; pulmonary; COPD

\section{Introduction}

Pulmonary rehabilitation (PR) is a "comprehensive intervention based on a thorough patient assessment followed by patient-tailored therapies that include, but are not limited to, exercise training, education, and behavior change, designed to improve the physical and psychological condition of people with chronic respiratory disease and to promote the longterm adherence to health-enhancing behaviors" [1]. It is an integral part of the management of patients with various respiratory diseases, along with other well-established modalities (e.g., oxygen, inhalers, and non-invasive ventilation). PR improves exercise capacity [1-3], limb muscle function [1,2,4], and health-related quality of life [1,2,5]. PR reduces symptom burden $[1,2,6]$, healthcare costs [1,2], hospitalizations [1,2], and other unplanned health care utilization [1,2]. Moreover, it has been shown to decrease mortality [7], as well as anxiety and depression [1,2]. Despite numerous benefits, PR as a resource remains 
underutilized. Less than 3\% of eligible candidates for PR attend one or more sessions after hospitalization [8-10]. Several barriers affect PR attendance and adherence. Enthusiasm of the referring physician, travel and distance to the PR center, caregiver knowledge and availability, smoking history, lack of trained manpower, and suboptimal funding are some of the most common barriers [1,11]. In a systematic review by Young et al., travel and transport were consistently reported as barriers across studies [12]. The current COVID-19 pandemic has significantly impacted clinic-based PR program enrollment and attendance, as people with chronic respiratory diseases are at an increased risk of severe COVID-19 disease [13], and many have been advised to stay at home and avoid in person contacts. This recommendation has led to a further widening in the already existing gap in care for those living with respiratory diseases. Emerging alternative models of PR delivery such as home-based PR, tele-rehabilitation, web-based PR, or hybrid models could help address these barriers and close the gap. In 2015, the American Thoracic Society (ATS) and European Respiratory Society (ERS) recommended further research to develop alternative delivery models to help improve access to PR. The ATS/ERS also stated that "adoption of alternative models for PR will require demonstration of comparable or greater clinical outcomes to those of traditional PR programs, as well as evaluation of safety and costeffectiveness, staff training and guideline development" [1]. Numerous studies have tested the feasibility, safety, and efficacy of these methods, but there is wide variability across studies and methods [14]. In this article, we reviewed studies to inform the question "Will remotely-based PR water down its effectiveness?"

\section{Methods}

We performed a 10-year literature review using PubMed, CINAHL, and Medline to identify relevant articles through 1 May 2021, for studies evaluating effectiveness of remotely-based PR. We reasoned that publications prior to 2011 would likely be less applicable, from a technology standpoint. The search strategy involved the keywords: tele-rehabilitation; pulmonary rehabilitation; rehabilitation; telehealth; chronic obstructive pulmonary disease (COPD); chronic lung disease; and COVID-19. The search was supplemented with articles from our personal files. We sought to identify Randomized Control Trials (RCTs), cohort studies, and case series. A total of 26 studies were identified, 10 of which were RCTs, 15 were cohort studies, and one was a case series (Table 1) (Figure 1).

Table 1. Studies evaluating tele-rehabilitation.

\begin{tabular}{|c|c|c|c|c|c|c|}
\hline Citation & $\begin{array}{c}\text { Study } \\
\text { Design/Purpose }\end{array}$ & $\begin{array}{c}\text { Patient } \\
\text { Disease/Sample } \\
\text { Size }\end{array}$ & $\begin{array}{l}\text { Rehab } \\
\text { Site }\end{array}$ & Intervention & Results & Adverse Events \\
\hline \multicolumn{7}{|c|}{ Tele-Rehabilitation vs. Conventional Pulmonary Rehab } \\
\hline $\begin{array}{c}\text { Layton et al., } 2021 \\
\text { [15] }\end{array}$ & $\begin{array}{l}\text { Cohort Study/ } \\
\text { Feasibility and } \\
\text { efficacy Study }\end{array}$ & $\begin{array}{l}\text { Cystic Fibrosis/ } \\
n=11 \text { vs. } n=8\end{array}$ & Home & $\begin{array}{l}\text { Smartphone } \\
\text { based } \\
\text { application }\end{array}$ & $\begin{array}{l}\text { Increased adherence } \\
\qquad(p=0.03)\end{array}$ & Muscle pain \\
\hline $\begin{array}{l}\text { Hansen et al., } \\
2020 \text { [16] }\end{array}$ & $\begin{array}{c}\mathrm{RCT} / \\
\text { Superiority trial }\end{array}$ & $\begin{array}{c}\text { COPD } / \\
n=67 \text { vs. } 67\end{array}$ & Home & $\begin{array}{l}\text { Real time } \\
\text { broadcast by } \\
\text { physiotherapist } \\
\text { and nurse }\end{array}$ & $\begin{array}{l}\text { No difference in 6MWT } \\
\text { between groups. } \\
\text { Higher rate of completion in } \\
\text { the tele rehabilitation group } \\
\qquad(p<0.01)\end{array}$ & $\begin{array}{c}2 \mathrm{AE}^{\prime} \mathrm{s} \text { in the } \\
\text { Conventional PR } \\
\text { group. }\end{array}$ \\
\hline $\begin{array}{l}\text { Rutkowski et al., } \\
2020 \text { [17] }\end{array}$ & $\begin{array}{c}\mathrm{RCT} / \\
\text { Superiority trial }\end{array}$ & $\begin{array}{c}\text { COPD/ } \\
n=106 \\
n=34 \\
\text { Conventional PR } \\
n=38 \\
\text { Conventional PR } \\
+ \text { VR } \\
n=34 \text { VR }\end{array}$ & Inpatient & $\begin{array}{l}\text { Virtual game } \\
\text { system } \\
\text { Conventional PR } \\
\text { with } \\
\text { physiotherapist }\end{array}$ & $\begin{array}{l}\text { PR+VR group was superior } \\
\text { to just PR group in Arm curl } \\
(p<0.003), \text { chair stand } \\
(p<0.008), \mathrm{Up} \text { and Go } \\
(p<0.000), \text { and 6MWT } \\
(p<0.011) \text { VR group } \\
\text { superior to PR in Arm curl } \\
(p<0.000), \text { chair stand } \\
(p<0.001) \text { and } 6 \mathrm{MWT} \\
(p<0.031)\end{array}$ & Not mentioned * \\
\hline
\end{tabular}


Table 1. Cont.

\begin{tabular}{|c|c|c|c|c|c|c|}
\hline Citation & $\begin{array}{c}\text { Study } \\
\text { Design/Purpose }\end{array}$ & $\begin{array}{c}\text { Patient } \\
\text { Disease/Sample } \\
\text { Size }\end{array}$ & $\begin{array}{l}\text { Rehab } \\
\text { Site }\end{array}$ & Intervention & Results & Adverse Events \\
\hline $\begin{array}{l}\text { Bernocchi et al., } \\
2018 \text { [18] }\end{array}$ & $\begin{array}{l}\text { RCT/Feasibility } \\
\text { and efficacy } \\
\text { Study }\end{array}$ & $\begin{array}{c}\text { COPD } / \\
n=56 \text { vs. } n=56\end{array}$ & Home & $\begin{array}{l}\text { Physiotherapist } \\
\text { weekly phone call }\end{array}$ & $\begin{array}{l}\text { Improvement in 6MWT } \\
\qquad(p<0.0040)\end{array}$ & None reported ${ }^{* *}$ \\
\hline $\begin{array}{l}\text { Horton et al., } \\
2018 \text { [19] }\end{array}$ & $\begin{array}{c}\text { RCT/ } \\
\text { Non-inferiority } \\
\text { trial }\end{array}$ & $\begin{array}{c}\text { COPD } / \\
n=145 \text { vs. } n=142\end{array}$ & Home & $\begin{array}{l}\text { Physiotherapist } \\
\text { weekly phone call }\end{array}$ & $\begin{array}{l}\text { No improvement in CRQ } \\
\text { dyspnea }(p=0.18)\end{array}$ & None reported \\
\hline $\begin{array}{l}\text { Vasilopoulou } \\
\text { et al., } 2017 \text { [20] }\end{array}$ & $\begin{array}{c}\text { RCT/Efficacy } \\
\text { Trial }\end{array}$ & $\begin{array}{l}\text { COPD } / \\
n=47 \text { vs. } n=50\end{array}$ & Home & $\begin{array}{l}\text { Recorded session } \\
\text { by } \\
\text { physiotherapist, } \\
\text { exercise scientist }\end{array}$ & $\begin{array}{c}\text { Home based PR and } \\
\text { Conventional PR decreased } \\
\text { COPD exacerbation and } \\
\text { hospitalization when } \\
\text { compared to } \\
\text { pharmacotherapy }(p<0.001)\end{array}$ & Not mentioned \\
\hline $\begin{array}{l}\text { Bourne et al., } \\
2017 \text { [21] }\end{array}$ & $\begin{array}{l}\mathrm{RCT} / \\
\text { Non-inferiority } \\
\text { trial }\end{array}$ & $n=64$ vs. $n=26$ & Home & $\begin{array}{l}\text { Pre-recorded } \\
\text { session by a } \\
\text { physiotherapist }\end{array}$ & $\begin{array}{l}\text { Online PR was non-inferior } \\
\text { to Conventional PR in } \\
6 \mathrm{MWT}(p=0.098) \text { and CAT } \\
(p=0.373)\end{array}$ & $\begin{array}{l}\text { Back pain and } \\
\text { muscular pain }\end{array}$ \\
\hline $\begin{array}{l}\text { Chaplin et al., } \\
2017 \text { [22] }\end{array}$ & $\begin{array}{l}\text { RCT / Feasibility } \\
\text { and efficacy trial }\end{array}$ & $\begin{array}{c}\text { COPD } / \\
n=51 \text { vs. } n=52\end{array}$ & Home & $\begin{array}{l}\text { Pre-recorded } \\
\text { session } \\
\text { physiotherapist }\end{array}$ & $\begin{array}{l}\text { No significant difference in } \\
\text { the CRQ dyspnea }(p>0.05) \text {, } \\
\text { ESWT }(p>0.05)\end{array}$ & Not mentioned \\
\hline $\begin{array}{l}\text { Paneroni et al., } \\
2015 \text { [23] }\end{array}$ & $\begin{array}{l}\text { Cohort Study/ } \\
\text { Feasibility and } \\
\text { Efficacy Study }\end{array}$ & $n=18$ vs. $n=18$ & Home & $\begin{array}{l}\text { Real time video } \\
\text { conferencing with } \\
\text { the } \\
\text { physiotherapist }\end{array}$ & $\begin{array}{l}\text { Improvement in physical } \\
\text { activity (steps per day) } \\
\quad(p<0.0002) \\
\text { No statistically significant } \\
\text { difference in 6MWT, } \\
\text { SGRQ or mMRC }\end{array}$ & None reported \\
\hline $\begin{array}{l}\text { Tabak et al., } \\
2014 \text { [24] }\end{array}$ & $\begin{array}{l}\text { Cohort Study/ } \\
\text { Feasibility and } \\
\text { Efficacy Study }\end{array}$ & $\begin{array}{c}\mathrm{COPD} / n=15 \mathrm{vs} . \\
n=14\end{array}$ & Home & $\begin{array}{l}\text { Pre-recorded } \\
\text { session by } \\
\text { physiotherapist }\end{array}$ & $\begin{array}{c}\text { Improvement in } \\
\text { mMRC scale }(p<0.03)\end{array}$ & Not mentioned \\
\hline $\begin{array}{l}\text { Stickland et al., } \\
2011[25]\end{array}$ & $\begin{array}{l}\text { Cohort Study/ } \\
\text { Efficacy Study }\end{array}$ & $n=147$ vs. $n=262$ & $\begin{array}{l}\text { Satellite } \\
\text { Center } \\
\text { under } \\
\text { super- } \\
\text { vision }\end{array}$ & $\begin{array}{l}\text { Real time video } \\
\text { conferencing with } \\
\text { respiratory } \\
\text { therapist }\end{array}$ & $\begin{array}{l}\text { Both telehealth PR and } \\
\text { Conventional PR showed } \\
\text { improvement in SGRQ } \\
\qquad(p<0.05)\end{array}$ & Not mentioned \\
\hline \multicolumn{7}{|c|}{ Tele-rehabilitation Alone (Pre vs. Post Intervention) } \\
\hline $\begin{array}{l}\text { Lewis et al., } \\
2021 \text { [26] }\end{array}$ & $\begin{array}{l}\text { Cohort Study/ } \\
\text { Efficacy and } \\
\text { Feasibility Study }\end{array}$ & $\begin{array}{l}\text { COPD/ } \\
n=17\end{array}$ & Home & $\begin{array}{l}\text { Physiotherapist } \\
\text { by real time video } \\
\text { conferencing }\end{array}$ & $\begin{array}{c}\text { Improvements in } 1 \text { min STS } \\
(p=0.004), \mathrm{GAD}(p=0.023), \\
\text { PHQ-9 }(p=0.029), \mathrm{CRQ} \\
\text { dyspnea }(p=0.001), \mathrm{CRQ} \\
\text { fatigue }(p=0.004), \mathrm{CRQ} \\
\text { emotion }(p=0.0002), \mathrm{CRQ} \\
\text { mastery }(p=0.001)\end{array}$ & None reported \\
\hline $\begin{array}{l}\text { Paneroni et al., } \\
2021 \text { [27] }\end{array}$ & $\begin{array}{l}\text { Cohort Study/ } \\
\text { Efficacy and } \\
\text { Feasibility Study }\end{array}$ & $\begin{array}{c}\text { COVID-19/ } \\
n=25\end{array}$ & Home & $\begin{array}{c}\text { Twice a week call } \\
\text { by a } \\
\text { physiotherapist }\end{array}$ & $\begin{array}{l}\text { Improvement in STS } \\
(p=0.003) \text { and 6MWT } \\
(p=0.0006)\end{array}$ & None Reported \\
\hline $\begin{array}{l}\text { Wootton et al., } \\
2020 \text { [28] }\end{array}$ & Case Series & $\begin{array}{c}\text { COVID-19/ } \\
n=3\end{array}$ & Home & $\begin{array}{l}\text { Weekly call by } \\
\text { physiotherapist }\end{array}$ & $\begin{array}{l}\text { Improvement in } 1 \text { min and } \\
5 \text { min STS }\end{array}$ & Not mentioned \\
\hline $\begin{array}{l}\text { Rassouli et al., } \\
\text { 2018 [29] }\end{array}$ & $\begin{array}{c}\text { Cohort } \\
\text { Study/Efficacy } \\
\text { and Feasibility } \\
\text { Study }\end{array}$ & $\mathrm{COPD} / n=34$ & Home & $\begin{array}{l}\text { Smartphone } \\
\text { application; } \\
\text { pre-recorded } \\
\text { videos }\end{array}$ & $\begin{array}{l}\text { Improvement in CAT scores } \\
\qquad(p=0.008) \\
\text { Improvement in CRQ } \\
\text { fatigue }(p<0.001), \text { mastery } \\
(p<0.001) \text { and emotion } \\
\quad(p<0.001) .\end{array}$ & Not mentioned \\
\hline $\begin{array}{l}\text { Zanaboni et al., } \\
2017 \text { [30] }\end{array}$ & $\begin{array}{l}\text { Cohort Study/ } \\
\text { Efficacy and } \\
\text { Feasibility study }\end{array}$ & $\begin{array}{l}\text { COPD/ } \\
n=10\end{array}$ & Home & $\begin{array}{l}\text { Real time video } \\
\text { conferencing with } \\
\text { Physiotherapist }\end{array}$ & $\begin{array}{l}\text { Improvement in 6MWT, } \\
\text { CAT }(p=0.022) \text { scores }\end{array}$ & Not mentioned \\
\hline $\begin{array}{l}\text { Hoaas et al., } \\
2016 \text { [31] }\end{array}$ & $\begin{array}{l}\text { Cohort Study/ } \\
\text { Efficacy and } \\
\text { Feasibility Study }\end{array}$ & $\mathrm{COPD} / n=10$ & Home & $\begin{array}{l}\text { Pre-recorded } \\
\text { session by } \\
\text { physiotherapist }\end{array}$ & $\begin{array}{l}\text { Decrease in physical activity } \\
\text { (Steps per day) } 1 \text { year after a } \\
\text { 2-year intervention } \\
(p=0.039)\end{array}$ & Not mentioned \\
\hline $\begin{array}{l}\text { Marquis et al., } \\
2014 \text { [32] }\end{array}$ & $\begin{array}{l}\text { Cohort Study/ } \\
\text { Efficacy and } \\
\text { Feasibility Study }\end{array}$ & $\begin{array}{l}\text { COPD/ } \\
n=26\end{array}$ & Home & $\begin{array}{c}\text { Combined } \\
\text { Real-time video } \\
\text { conferencing by } \\
\text { physiotherapists } \\
\text { and unsupervised } \\
\text { sessions }\end{array}$ & $\begin{array}{c}\text { Improvement in 6MWT } \\
(p<0.001), \text { CET }(p=0.003) \\
\text { and CRQ }(p<0.001) \text { at } 8 \\
\text { weeks but not sustained } \\
\text { until } \\
\quad 24 \text {-week follow-up }\end{array}$ & Not mentioned \\
\hline
\end{tabular}


Table 1. Cont.

\begin{tabular}{|c|c|c|c|c|c|c|}
\hline Citation & $\begin{array}{c}\text { Study } \\
\text { Design/Purpose }\end{array}$ & $\begin{array}{c}\text { Patient } \\
\text { Disease/Sample } \\
\text { Size }\end{array}$ & $\begin{array}{l}\text { Rehab } \\
\text { Site }\end{array}$ & Intervention & Results & Adverse Events \\
\hline $\begin{array}{l}\text { Albores et al., } \\
2013 \text { [33] }\end{array}$ & $\begin{array}{l}\text { Cohort Study/ } \\
\text { Efficacy and } \\
\text { Feasibility Study }\end{array}$ & $\begin{array}{l}\text { COPD/ } \\
n=25\end{array}$ & Home & $\begin{array}{l}\text { Virtual Game } \\
\text { system }\end{array}$ & $\begin{array}{c}\text { Improvement in ESWT } \\
(p=0.005) \text {, arm-lift } \\
(p=0.03), \text { sit to stand } \\
\text { repetitions }(p=0.03) \text { and } \\
\text { CRQ emotion scores } \\
(p=0.02)\end{array}$ & Not mentioned \\
\hline $\begin{array}{l}\text { Holland et al., } \\
2013[34]\end{array}$ & $\begin{array}{l}\text { Cohort Study/ } \\
\text { Feasibility Study }\end{array}$ & $\begin{array}{l}\text { COPD/ } \\
n=8\end{array}$ & Home & $\begin{array}{l}\text { Real-time } \\
\text { videoconferenc- } \\
\text { ing with } \\
\text { physiotherapist }\end{array}$ & $\begin{array}{l}\text { Improvement in 6MWT, } \\
\text { CRQ score }\end{array}$ & $\begin{array}{c}\text { Minor adverse } \\
\text { events were } \\
\text { desaturation < } \\
88 \%(n=1) \& \\
\text { heart rate }>150 \\
\text { BPM }(n=1)\end{array}$ \\
\hline $\begin{array}{l}\text { Wardini et al., } \\
2013 \text { [35] }\end{array}$ & $\begin{array}{l}\text { Cohort Study/ } \\
\text { Feasibility Study }\end{array}$ & $\begin{array}{l}\text { COPD/ } \\
n=32\end{array}$ & $\begin{array}{l}\text { Inpatient } \\
\text { con- } \\
\text { ven- } \\
\text { tional }+ \\
\text { virtual }\end{array}$ & $\begin{array}{l}\text { Virtual game } \\
\text { system }\end{array}$ & $\begin{array}{l}\text { Increased enjoyment using } \\
\text { VAS } \\
\text { Increased adherence }\end{array}$ & None reported \\
\hline $\begin{array}{l}\text { Tousignant et al., } \\
2012[36]\end{array}$ & $\begin{array}{l}\text { Cohort Study/ } \\
\text { Feasibility Study }\end{array}$ & $\mathrm{COPD} / n=3$ & Home & $\begin{array}{l}\text { Real time } \\
\text { videoconferenc- } \\
\text { ing with } \\
\text { physiotherapist }\end{array}$ & $\begin{array}{l}\text { Improvement in } 6 \mathrm{MWT} \text { for } \\
2 \text { out of } 3 \text { participants }\end{array}$ & Not mentioned \\
\hline \multicolumn{7}{|c|}{ Tele-rehabilitation vs. No Rehabilitation } \\
\hline $\begin{array}{l}\text { Gonzalez-Gerez } \\
\text { et al., } 2021 \text { [37] }\end{array}$ & $\begin{array}{c}\text { RCT/ } \\
\text { Feasibility and } \\
\text { Efficacy Trial }\end{array}$ & $\begin{array}{c}\text { COVID-19/ } \\
n=19 \text { vs. } n=19\end{array}$ & Home & $\begin{array}{l}\text { Twice weekly } \\
\text { calls by } \\
\text { physiotherapist }\end{array}$ & $\begin{array}{c}\text { Improvement in 6MWT } \\
(p<0.001) \text { and dyspnea } \\
\text { perception using Borg scale } \\
(p<0.001)\end{array}$ & None Reported \\
\hline $\begin{array}{l}\text { Li et al., } \\
2021[38]\end{array}$ & $\begin{array}{c}\text { RCT/ } \\
\text { Efficacy Trial }\end{array}$ & $\begin{array}{c}\text { COVID- } 19 / \\
n=59 \text { vs. } n=61\end{array}$ & Home & $\begin{array}{l}\text { Smartphone- } \\
\text { based } \\
\text { application }\end{array}$ & $\begin{array}{c}\text { Improvement in 6MWT } \\
(p<0.001), \text { mMRC } \\
(p<0.001), \text { LMS }(p<0.001) \\
\text { and SF-12 PCS }(p<0.001)\end{array}$ & None reported \\
\hline $\begin{array}{l}\text { Bhatt et al., } \\
2019 \text { [39] }\end{array}$ & $\begin{array}{l}\text { Cohort Study/ } \\
\text { Feasibility and } \\
\text { Efficacy Study }\end{array}$ & $\begin{array}{c}\text { COPD } / \\
n=80 \text { vs. } n=160\end{array}$ & Home & $\begin{array}{l}\text { Physiotherapist } \\
\text { by real-time video } \\
\text { conferencing }\end{array}$ & $\begin{array}{l}\text { Decreased 30-day all-cause } \\
\text { mortality }(p=0.013) \text { and } \\
\text { readmissions due to } \\
\text { AECOPD }(p=0.04)\end{array}$ & None reported \\
\hline $\begin{array}{l}\text { Tsai, } \\
2017[40]\end{array}$ & $\begin{array}{c}\text { RCT / } \\
\text { Efficacy Trial }\end{array}$ & $\begin{array}{c}\text { COPD } / \\
n=37 \text { vs. } n=37\end{array}$ & Home & $\begin{array}{l}\text { Real-time } \\
\text { broadcast by } \\
\text { physiotherapist }\end{array}$ & $\begin{array}{c}\text { Improvement in ESWT } \\
(p<0.001) \text {, self-efficacy } \\
(p<0.007) \text { and CRQ } \\
(p=0.07)\end{array}$ & Not mentioned \\
\hline
\end{tabular}

AE: Adverse Event; AECOPD: Acute Exacerbation of Chronic Obstructive Pulmonary Disease; CAT: COPD Assessment Test; CET: Constant work rate Exercise Test; COPD: Chronic Obstructive Pulmonary Disease; CRQ: Chronic Respiratory Questionnaire; ESWT: Endurance Shuttle Walk Test; EQ-VAS: EuroQol Visual Analog Scale; GAD: Generalized Anxiety Disorder; MRC: Medical Research Council; mMRC: Modified Medical Research Council; LMS: Lower limb muscle Strength; PCS: Physical Component Score; PHQ-9: Primary Health Questionnaire-9; PR: Pulmonary Rehabilitation; RCT: Randomized Control Trial; SF-12: Short Form Health Survey-12; SGRQ: St George's Respiratory Questionnaire; STS: Sit To Stand; VR: Virtual Reality; 6MWT: 6 Minute Walk Test. * Not mentioned-Studies did not look for adverse events. ${ }^{* *}$ None reported-Studies reported the absence of adverse events. 


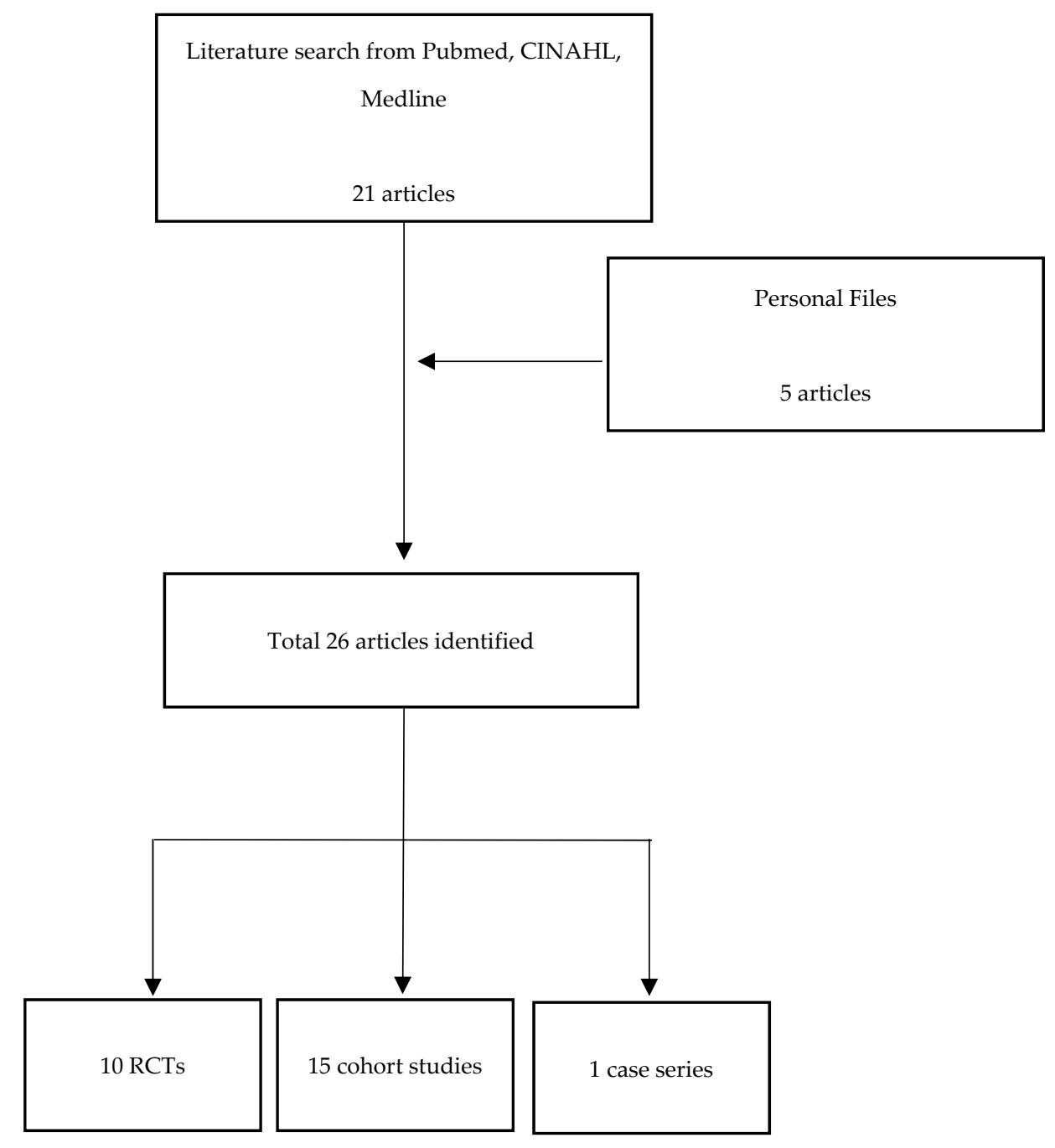

Figure 1. Flow diagram illustrating selection of eligible studies.

\section{Results}

Out of the total 26 studies conducted between 2011 and 2021; 15 (58\%) were cohort studies, 10 (38\%) were RCTs and one (4\%) was a case series (Table 1). Twenty-one (81\%) of the studies included participants with COPD [16-26,29-36,39,40], four (15\%) with COVID$19[27,28,37,38]$ and one (4\%) with cystic fibrosis [15].

\subsection{Tele-Rehabilitation vs. Conventional Pulmonary Rehab}

Eleven studies compared tele-rehabilitation to conventional clinic-based PR (Table 2), seven of which were RCTs [16-22] and 4 were cohort studies [15,23-25]. The RCTs included a total of $n=979$ study participants with $n=477$ participants receiving traditional PR while 502 received a tele-rehabilitation-based intervention.

Modes of Tele-rehabilitation: A variety of modes of tele-rehabilitation were used, including real-time broadcast by a healthcare provider (HCP) to the participant's home $[16,23]$ and to a satellite center near the participant's home [25]. These were standardized group sessions with durations varying from $30 \mathrm{~min}$ [16], to $60 \mathrm{~min}$ [23] and to $2 \mathrm{~h} \mathrm{[25].} \mathrm{Others}$ used an online platform with pre-recorded sessions [20-22,24] or a smartphone-based application [15]. The sessions were individualized and were performed at the patients' convenience. Patients' progress was monitored online using questionnaires $[15,20,21,24]$. In all the studies there was contact with the research team or HCP via email or phone to ensure adequate patient progress or to answer queries [15,20-22,24]. Unsupervised home-based 
rehabilitation with standardized weekly phone calls by a HCP to monitor patient progress was used in two studies [18,19]. A virtual gaming system with and without conventional PR was used in one study [17].

Outcomes: Outcomes from these studies found that, compared with conventional clinic-based PR, the tele-rehabilitation group demonstrated the following improvements: better adherence [15], upper extremity strength as measured by the arm curl test [17], leg strength as measured by the Chair Stand Test [17], functional behavior as measured by the Up and Go Test [17] and physical activity (steps per day) [23]. Distance outcomes from the 6 min walk test (6MWT) varied from increased [17,18], not different $[16,23]$, and not inferior to conventional clinic-based PR [21]. Dyspnea as measured by the Chronic Respiratory Questionnaire (CRQ) was not different in one study [22] when compared to conventional PR; however, in another study, it was inferior [19]. Improvement was seen in the Endurance Shuttle Walk test (ESWT) and were not different from improvements seen in traditional PR [22]. Outcomes from the Modified Medical Research Council (mMRC) were improved [24] and not different [23] from those found with conventional PR. When telerehabilitation was compared to conventional PR, St. George's Respiratory Questionnaire (SGRQ) improved in both groups [25], and the differences between groups were not different $[23,25]$. When compared to pharmacotherapy alone, both home-based PR and conventional PR decreased COPD exacerbations and hospitalization [25].

Table 2. Tele-rehabilitation vs. Conventional Pulmonary Rehabilitation.

\begin{tabular}{|c|c|c|c|c|}
\hline Outcome & Improved $^{\mathrm{a}}$ & Inferior $^{b}$ & Not Different ${ }^{c}$ & Not Inferior ${ }^{d}$ \\
\hline 6MWT & $\begin{array}{l}\text { Rutkowski et al. [17], } \\
\text { Bernocchi et al. [18] }\end{array}$ & & $\begin{array}{l}\text { Hansen et al. [16], } \\
\text { Paneroni et al. [23] }\end{array}$ & Bourne et al. [21] \\
\hline CRQ dyspnea & & Horton et al. [19] & Chaplin et al. [22] & \\
\hline ESWT & & & Chaplin et al. [22] & \\
\hline mMRC & Tabak et al. [24] & & Paneroni et al. [23] & \\
\hline SGRQ & $\begin{array}{l}\text { Stickland et al. [25] } \\
\text { - so did traditional }\end{array}$ & & Paneroni et al. [23] & \\
\hline CAT & & & & Bourne et al. [21] \\
\hline Arm Curl & Rutkowski et al. [17] & & & \\
\hline Chair Stand & Rutkowski et al. [17] & & & \\
\hline Up and Go & Rutkowski et al. [17] & & & \\
\hline $\begin{array}{l}\text { Physical Activity } \\
\text { (steps per day) }\end{array}$ & Paneroni et al. [23] & & & \\
\hline \multicolumn{5}{|c|}{$\begin{array}{l}\text { CRQ: Chronic Respiratory Disease; ESWT: Endurance Shuttle Walk Test; CAT: COPD Assessment Test; HADS: Hospital Anxiety and } \\
\text { Depression Scale; mMRC: Modified Medical Research Council; SGRQ: St. George's Respiratory Questionnaire; } 6 \text { MWT: } 6 \text { Minute Walk } \\
\text { Test. }{ }^{\mathbf{a}} \text { Statistically significant improvement found in both groups. }{ }^{\mathbf{b}} \text { In this non-inferiority study tele-rehab was found to be inferior to } \\
\text { conventional PR. }^{\mathbf{c}} \text { No statistical difference in outcomes between intervention and control group. }{ }^{\mathbf{d}} \text { Non inferiority threshold reached in the } \\
\text { non-inferiority RCT. }\end{array}$} \\
\hline
\end{tabular}

\subsection{Tele-Rehabilitation Only (Pre-Intervention vs. Post Intervention)}

Eleven studies evaluated tele-rehabilitation using the patient's baseline status as control (Table 3), ten of which were cohort studies [26,27,29-36] and one was a case series [28]. Seven studies were both efficacy and feasibility trials, whereas four of them evaluated feasibility alone [28,34-36]. A total of $n=187$ participants were included in these studies.

Modes of Tele-Rehabilitation: Studies involving tele-rehabilitation without a comparison group used a variety of interventional modes including real-time broadcast by an HCP to the participant's home $[26,30,32,34,36]$. Zanaboni et al. [30] used an individualized treadmill program whereas standardized group sessions were used in other studies $[26,32,34,36]$. Unsupervised pre-recorded videos on a smartphone application and online platform were used in two studies $[29,31]$. Patients in these two studies were expected to participate daily 
in one study [29] and three times a week in another [31]. Virtual gaming was used in two studies [33,35]. One study used virtual gaming in addition to conventional PR [35] three times a week, whereas a virtual gaming system alone was used in the other study [33]. Unsupervised weekly phone calls to remotely monitor patient progress were used in two studies $[27,28]$.

Table 3. Tele-rehabilitation (Pre vs. Post Intervention).

\begin{tabular}{|c|c|c|}
\hline Outcome & Improved & No Improvement \\
\hline $6 \mathrm{MWT}$ & $\begin{array}{l}\text { Paneroni et al. [27] } \\
\text { Zanaboni et al. [30] } \\
\text { Marquis et al. [32] } \\
\text { Holland et al. [34] } \\
\text { Tousignant et al. [36] }\end{array}$ & \\
\hline CRQ dyspnea & $\begin{array}{c}\text { Marquis et al. [32] } \\
\text { Holland et al. [34] } \\
\text { Lewis et al. [26] }\end{array}$ & \\
\hline ESWT & Albores et al. [33] & \\
\hline CAT & $\begin{array}{l}\text { Zanaboni et al. [30] } \\
\text { Rassouli et al. [29] }\end{array}$ & \\
\hline Arm Curl & Albores et al. [33] & \\
\hline Chair Stand & $\begin{array}{l}\text { Lewis et al. [26] } \\
\text { Paneroni et al. [27] } \\
\text { Wootton et al. [28] } \\
\text { Albores et al. [33] }\end{array}$ & \\
\hline $\begin{array}{l}\text { Physical Activity } \\
\text { (steps per day) }\end{array}$ & & Hoaas et al. [31] \\
\hline
\end{tabular}

CRQ: Chronic Respiratory Questionnaire; ESWT: Endurance Shuttle Walk Test; CAT: COPD Assessment Test; 6MWT: 6 Minute Walk Test.

Outcomes: All 11 studies found that tele-rehabilitation was feasible [26-36] (Table 3). When comparing study endpoints before and after the intervention, the tele-rehabilitation group had better adherence (defined as $>50 \%$ sessions completed) [35], improved upper extremity strength as measured by the arm lift test [33], and increased leg strength as measured by the Sit to Stand Test [26-28,33]. There was also an improvement in both the 6MWT [27,30,32,34,36] and ESWT [33]. Dyspnea, as measured by the CRQ [26,32,34] and CAT scores [29,30], were improved after tele-rehabilitation. There was also an improvement in Health-Related Quality of Life (HRQoL) using the CRQ scores [26,29,33]. Hoaas et al. (2016), examined the provision of equipment for self-management and unsupervised home exercise for 1 year after an intervention. They found a decrease in physical activity (steps per day) suggesting that the availability of equipment might not be sufficient to maintain physical activity levels post-rehab completion [31].

\subsection{Tele-Rehabilitation vs. No Rehabilitation}

Four studies (Table 4) compared tele-rehabilitation to no rehabilitation [37-40], three of which were RCTs [37,38,40], and one was a cohort study [39]. The RCTs included a total of $n=234$ participants, of which $n=115$ received a tele-rehabilitation-based intervention. The lone cohort study was both a feasibility and an efficacy study [39].

Modes of Tele-Rehabilitation: When comparing tele-rehabilitation to no rehabilitation, the various modes of delivery included real-time broadcast by an HCP to the participant's home [39,40], pre-recorded sessions on a smartphone-based application [38], and twice weekly phone calls to otherwise unsupervised study participants [37]. Among studies utilizing real time broadcast, one study [39] used individualized sessions three times a week for 12 weeks, whereas group sessions three times a week for 8 weeks was used in the other [40]. Another study used a smartphone-based application which provided weekly 
teleconsultations in addition to 3-4 unsupervised sessions per week [38]. Unsupervised breathing exercises for 7 days with biweekly check-ins using telephone calls was used in one study [37].

Table 4. Tele-rehabilitation vs. No Rehabilitation.

\begin{tabular}{ccc}
\hline Outcome & Improved & Not Different \\
\hline 6MWT & $\begin{array}{c}\text { Gonzalez-Gerez et al. [37] } \\
\text { Li et al. [38] }\end{array}$ & Tsai et al. [40] \\
\hline Borg dyspnea scale & Gonzalez-Gerez et al. [37] & \\
\hline ESWT & Tsai et al. [40] & \\
\hline mMRC & Li et al. [38] \\
Tsai et al. [40] & Li et al. [38] \\
\hline Static Squat Test & Tsai et al. [40] & \\
\hline HADS & Tsai et al. [40] \\
\hline CRQ & Bhatt et al. [39] \\
\hline 30-day all-cause mortality & CRQ: Chronic Respiratory Questionnaire; ESWT: Endurance Shuttle Walk Test, CAT: COPD Assessment Test; \\
HADS: Hospital Anxiety and Depression Scale; mMRC: Modified Medical Research Council; 6MWT: 6 Minute \\
Walk Test.
\end{tabular}

Outcomes: Tele-rehabilitation compared with no rehabilitation was found to be feasible $[37,39]$ and efficacious $[38,40]$. These studies showed that compared to no rehabilitation intervention, the tele-rehabilitation group showed improvements in the following areas: perceived dyspnea using the Borg scale [37], lower limb muscle strength using a static squat test [38], HRQoL using Short Form Health Survey-12 (SF-12), Physical Component Score (PCS) and CRQ scores, [38,40], and both the 6MWT and ESWT [37,38,40]. Psychological benefit was also seen using the Hospital Anxiety and Depression Scale (HADS) score [40]. Decreased mortality and readmission rates due to COPD exacerbations were also seen in this group [39].

\section{Discussion}

Remotely-based PR is a feasible option for people living with chronic lung diseases, especially those with COPD [16-26,29-36,39,40]. It may also be an option for patients recovering from the remote effects of COVID-19 [27,28,37,38]. Despite the different technologies used for telecommunication, these studies support tele-rehabilitation as a feasible option. In all the studies, both an educational component and a physical activity component was present [15-40].

When compared to no rehabilitation, tele-rehabilitation was effective across studies. It consistently showed statistically significant improvements in exercise capacity $[17,18,22$, $26-28,30,32-34,36-38,40]$ and dyspnea perception $[19,22,24-26,32,34,37,38,40]$ as well as a decreased 30-day mortality and readmission rates for acute exacerbations due to COPD [39]. This makes tele-rehabilitation an attractive option for patients who have significant barriers to attending conventional, clinic-based PR. When compared to conventional PR, telerehabilitation was found to be equally effective in the majority of studies. Multiple noninferiority trials showed no significant difference in outcomes between groups, and instead found tele-rehabilitation to be as effective as conventional PR [19,21]. Hansen et al. (2020), failed to show that telehealth was superior to conventional PR, however participants in the tele-rehabilitation group had higher completion rates [16].

Despite several studies showing feasibility and non-inferiority to conventional PR, the acceptance and implementation of remotely based tele-rehabilitation has been slow and highly variable over the years [14]. The current COVID-19 pandemic has greatly impacted conventional, in-person rehabilitation enrollment and attendance. This has re-focused the spotlight on tele-rehabilitation as an option for patients with chronic lung disease. However, 
the studies are heterogeneous in design with small sample sizes, use inconsistent outcome measures, and most importantly, use a wide variety of technologies [41]. Another major hurdle that has likely reduced the uptake of remote-based PR is the paucity of data regarding its cost effectiveness. Tele-rehabilitation involves using complex technology and equipment to monitor patients, and it also needs trained manpower-which can be expensive. Many insurers are willing to pay for conventional PR but not tele-rehabilitation [42]. Wide-spread acceptance of tele-rehabilitation in the healthcare system across the US is hampered by regulations and restrictions by state governments and policies of insurers [43]. Limitations such as potential for injury, digital and health literacy, and lack of appropriate device/internet connection remain. It is important to understand when tele-rehabilitation is safe and which subtype of patient benefits the most from tele-rehabilitation. Small studies have shown that tele-rehabilitation is safe with no major adverse events, [15,18,19,21,23,26,27,34,35,37,39], but large studies are lacking. The majority of the literature focuses on patients with COPD, thus making it difficult to extrapolate benefits to other lung diseases [44]. Challenges with digital literacy and familiarity are encountered especially in elderly frail patients. In a study by Chaplin et al. [22], high dropout rates were seen in the tele-rehabilitation group with technological challenges being the major reason. Investigators were required to modify their platform according to patient feedback.

\section{Conclusions}

The existing data does not show that remotely based PR will water down the effectiveness of conventional PR. Use of remotely based PR is a feasible and effective option to deliver PR, especially for patients with significant barriers to conventional clinic-based PR. Additional, well-conducted RCTs are needed to answer the questions regarding its efficacy, safety, cost-effectiveness and who will derive the maximum benefit among patients with COPD and other lung diseases.

Author Contributions: H.R.: Literature search, manuscript draft, final review and editing; S.D.C.: Literature search, manuscript draft, critical review and editing; S.M.F.: Manuscript draft, critical review and final editing; J.A.O.: Literature search, manuscript draft, critical review and editing. All authors have read and agreed to the published version of the manuscript.

Funding: Dr. Flynn's contribution to this research was funded in part by National Institutes of Health (NIH)-National Heart Lung and Blood Institute (NHLBI), grant number R44HL137502.

Institutional Review Board Statement: Not applicable.

Informed Consent Statement: Not applicable.

Data Availability Statement: Not applicable.

Conflicts of Interest: Sheryl Flynn is the CEO of Blue Marble Health and could benefit financially from the sale of products developed by Blue Marble Health. Sheryl Flynn is the PI of an NIH grant (R44HL137502) that is evaluating the feasibility of a digital pulmonary rehabilitation platform developed by her company.

\section{References}

1. Rochester, C.L.; Vogiatzis, I.; Holland, A.E.; Lareau, S.C.; Marciniuk, D.D.; Puhan, M.A.; Masefield, S.C.; Casaburi, R.; Clini, E. An Official American Thoracic Society/European Respiratory Society Policy Statement: Enhancing Implementation, Use, and Delivery of Pulmonary Rehabilitation. Am. J. Respir. Crit. Care Med. 2015, 192, 1373-1386. [CrossRef] [PubMed]

2. $\quad$ Spruit, M.A.; Singh, S.J.; Garvey, C.; ZuWallack, R.; Nici, L.; Rochester, C.; Hill, K.; Holland, A.E.; Lareau, S.C.; Man, W.D.C.; et al. An official American Thoracic Society/European Respiratory Society statement: Key concepts and advances in pulmonary rehabilitation. Am. J. Respir. Crit. Care Med. 2013, 188, e13-e64. [CrossRef]

3. Bolton, C.E.; Bevan-Smith, E.F.; Blakey, J.D.; Crowe, P.; Elkin, S.L.; Garrod, R.; Greening, N.J.; Heslop, K.; Hull, J.H.; Man, W.D.-C.; et al. British Thoracic Society guideline on pulmonary rehabilitation in adults. Thorax 2013, 68 (Suppl. 2), ii1-ii30. [CrossRef]

4. Maltais, F.; Decramer, M.; Casaburi, R.; Barreiro, E.; Burelle, Y.; Debigare, R.; Dekhuijzen, P.R.; Franssen, F.; Gayan-Ramirez, G.; Gea, J.; et al. An official American Thoracic Society/European Respiratory Society statement: Update on limb muscle dysfunction in chronic obstructive pulmonary disease. Am. J. Respir. Crit. Care Med. 2014, 189, e15-e62. [CrossRef] 
5. $\quad$ Lacasse, Y.; Goldstein, R.; Lasserson, T.J.; Martin, S. Pulmonary rehabilitation for chronic obstructive pulmonary disease. Cochrane Database Syst. Rev. 2006, 2, CD003793.

6. Laviolette, L.; Bourbeau, J.; Bernard, S.; Lacasse, Y.; Pepin, V.; Breton, M.J.; Baltzan, M.; Rouleau, M.; Maltais, F. Assessing the impact of pulmonary rehabilitation on functional status in COPD. Thorax 2008, 63, 115-121.

7. Lindenauer, P.K.; Stefan, M.S.; Pekow, P.S.; Mazor, K.M.; Priya, A.; Spitzer, K.A.; Lagu, T.C.; Pack, Q.R.; Pinto-Plata, V.M.; ZuWallack, R. Association Between Initiation of Pulmonary Rehabilitation After Hospitalization for COPD and 1-Year Survival Among Medicare Beneficiaries. JAMA. 2020, 323, 1813-1823.

8. Spitzer, K.A.; Stefan, M.S.; Priya, A.; Pack, Q.R.; Pekow, P.S.; Lagu, T.; Pinto-Plata, V.M.; ZuWallack, R.L.; Lindenauer, P.K. Participation in Pulmonary Rehabilitation after Hospitalization for Chronic Obstructive Pulmonary Disease among Medicare Beneficiaries. Ann. Am. Thorac. Soc. 2019, 16, 99-106. [PubMed]

9. Vercammen-Grandjean, C.; Schopfer, D.W.; Zhang, N.; Whooley, M.A. Participation in Pulmonary Rehabilitation by Veterans Health Administration and Medicare Beneficiaries After Hospitalization for Chronic Obstructive Pulmonary Disease. J. Cardiopulm. Rehabil. Prev. 2018, 38, 406-410.

10. Nishi, S.P.; Zhang, W.; Kuo, Y.F.; Sharma, G. Pulmonary Rehabilitation Utilization in Older Adults With Chronic Obstructive Pulmonary Disease, 2003 to 2012. J. Cardiopulm. Rehabil. Prev. 2016, 36, 375-382. [CrossRef]

11. Hayton, C.; Clark, A.; Olive, S.; Browne, P.; Galey, P.; Knights, E.; Staunton, L.; Jones, A.; Coombes, E.; Wilson, A.M. Barriers to pulmonary rehabilitation: Characteristics that predict patient attendance and adherence. Respir. Med. 2013, 107, $401-407$. [CrossRef]

12. Young, P.; Dewse, M.; Fergusson, W.; Kolbe, J. Respiratory rehabilitation in chronic obstructive pulmonary disease: Predictors of nonadherence. Eur. Respir. J. 1999, 13, 855-859. [CrossRef]

13. Aveyard, P.; Gao, M.; Lindson, N.; Hartmann-Boyce, J.; Watkinson, P.; Young, D.; Coupland, C.A.C.; Tan, P.S.; Clift, A.K.; Harrison, D.; et al. Association between pre-existing respiratory disease and its treatment, and severe COVID-19: A population cohort study. Lancet Respir. Med. 2021, 9, 909-923. [CrossRef]

14. Holland, A.E.; Cox, N.S.; Houchen-Wolloff, L.; Rochester, C.L.; Garvey, C.; ZuWallack, R.; Nici, L.; Limberg, T.; Lareau, S.C.; Yawn, B.P.; et al. Defining Modern Pulmonary Rehabilitation. An Official American Thoracic Society Workshop Report. Ann. Am. Thorac. Soc. 2021, 18, e12-e29. [CrossRef]

15. Layton, A.M.; Irwin, A.M.; Mihalik, E.C.; Fleisch, E.; Keating, C.L.; DiMango, E.A.; Shah, L.; Arcasoy, S.M. Telerehabilitation Using Fitness Application in Patients with Severe Cystic Fibrosis Awaiting Lung Transplant: A Pilot Study. Int. J. Telemed. Appl. 2021, 2021, 6641853. [CrossRef] [PubMed]

16. Hansen, H.; Bieler, T.; Beyer, N.; Kallemose, T.; Wilcke, J.T.; Ostergaard, L.M.; Andeassen, H.F.; Martinez, G.; Lavesen, M.; Frølich, A.; et al. Supervised pulmonary tele-rehabilitation versus pulmonary rehabilitation in severe COPD: A randomised multicentre trial. Thorax 2020, 75, 413-421. [CrossRef] [PubMed]

17. Rutkowski, S.; Rutkowska, A.; Kiper, P.; Jastrzebski, D.; Racheniuk, H.; Turolla, A.; Szczegielniak, J.; Casaburi, R. Virtual Reality Rehabilitation in Patients with Chronic Obstructive Pulmonary Disease: A Randomized Controlled Trial. Int. J. Chron. Obstruct. Pulmon. Dis. 2020, 15, 117-124. [CrossRef] [PubMed]

18. Bernocchi, P.; Vitacca, M.; La Rovere, M.T.; Volterrani, M.; Galli, T.; Baratti, D.; Paneroni, M.; Campolongo, G.; Sposato, B.; Scalvini, S. Home-based telerehabilitation in older patients with chronic obstructive pulmonary disease and heart failure: A randomised controlled trial. Age Ageing 2018, 47, 82-88. [CrossRef]

19. Horton, E.J.; Mitchell, K.E.; Johnson-Warrington, V.; Apps, L.D.; Sewell, L.; Morgan, M.; Taylor, R.S.; Singh, S.J. Comparison of a structured home-based rehabilitation programme with conventional supervised pulmonary rehabilitation: A randomised non-inferiority trial. Thorax 2018, 73, 29-36. [CrossRef]

20. Vasilopoulou, M.; Papaioannou, A.I.; Kaltsakas, G.; Louvaris, Z.; Chynkiamis, N.; Spetsioti, S.; Kortianou, E.; Genimata, S.A.; Palamidas, A.; Kostikas, K.; et al. Home-based maintenance tele-rehabilitation reduces the risk for acute exacerbations of COPD, hospitalisations and emergency department visits. Eur. Respir. J. 2017, 49, 1602129. [CrossRef]

21. Bourne, S.; DeVos, R.; North, M.; Chauhan, A.; Green, B.; Brown, T.; Cornelius, V.; Wilkinson, T. Online versus face-to-face pulmonary rehabilitation for patients with chronic obstructive pulmonary disease: Randomised controlled trial. BMJ Open 2017, 7, e014580. [CrossRef]

22. Chaplin, E.; Hewitt, S.; Apps, L.; Bankart, J.; Pulikottil-Jacob, R.; Boyce, S.; Morgan, M.; Williams, J.; Singh, S. Interactive web-based pulmonary rehabilitation programme: A randomised controlled feasibility trial. BMJ Open 2017, 7, e013682. [CrossRef] [PubMed]

23. Paneroni, M.; Colombo, F.; Papalia, A.; Colitta, A.; Borghi, G.; Saleri, M.; Cabiaglia, A.; Azzalini, E.; Vitacca, M. Is Telerehabilitation a Safe and Viable Option for Patients with COPD? A Feasibility Study. COPD 2015, 12, 217-225. [CrossRef] [PubMed]

24. Tabak, M.; Brusse-Keizer, M.; van der Valk, P.; Hermens, H.; Vollenbroek-Hutten, M. A telehealth program for self-management of COPD exacerbations and promotion of an active lifestyle: A pilot randomized controlled trial. Int. J. Chron. Obstruct. Pulmon. Dis. 2014, 9, 935-944. [CrossRef]

25. Stickland, M.; Jourdain, T.; Wong, E.Y.; Rodgers, W.M.; Jendzjowsky, N.G.; Macdonald, G.F. Using Telehealth technology to deliver pulmonary rehabilitation in chronic obstructive pulmonary disease patients. Can. Respir. J. 2011, 18, 216-220. [CrossRef] [PubMed] 
26. Lewis, A.; Knight, E.; Bland, M.; Middleton, J.; Mitchell, E.; McCrum, K.; Conway, J.; Bevan-Smith, E. Feasibility of an online platform delivery of pulmonary rehabilitation for individuals with chronic respiratory disease. BMJ Open Respir. Res. 2021, 8, e000880. [CrossRef] [PubMed]

27. Paneroni, M.; Vitacca, M.; Bernocchi, P.; Bertacchini, L.; Scalvini, S. Feasibility of tele-rehabilitation in survivors of COVID-19 pneumonia. Pulmonology 2021. [CrossRef]

28. Wootton, S.L.; King, M.; Alison, J.A.; Mahadev, S.; Chan, A.S.L. COVID-19 rehabilitation delivered via a telehealth pulmonary rehabilitation model: A case series. Respirol. Case Rep. 2020, 8, e00669. [CrossRef]

29. Rassouli, F.; Boutellier, D.; Duss, J.; Huber, S.; Brutsche, M.H. Digitalizing multidisciplinary pulmonary rehabilitation in COPD with a smartphone application: An international observational pilot study. Int. J. Chron. Obstruct. Pulmon. Dis. 2018, 13, 3831-3836. [CrossRef]

30. Zanaboni, P.; Hoaas, H.; Aaroen Lien, L.; Hjalmarsen, A.; Wootton, R. Long-term exercise maintenance in COPD via telerehabilitation: A two-year pilot study. J. Telemed. Telecare 2017, 23, 74-82. [CrossRef]

31. Hoaas, H.; Morseth, B.; Holland, A.E.; Zanaboni, P. Are Physical activity and Benefits Maintained After Long-Term Telerehabilitation in COPD? Int. J. Telerehabilit. 2016, 8, 39-48. [CrossRef]

32. Marquis, N.; Larivee, P.; Dubois, M.F.; Tousignant, M. Are improvements maintained after in-home pulmonary telerehabilitation for patients with chronic obstructive pulmonary disease? Int. J. Telerehabilt. 2014, 6, 21-30. [CrossRef]

33. Albores, J.; Marolda, C.; Haggerty, M.; Gerstenhaber, B.; Zuwallack, R. The use of a home exercise program based on a computer system in patients with chronic obstructive pulmonary disease. J. Cardiopulm. Rehabilt. Prev. 2013, 33, 47-52. [CrossRef]

34. Holland, A.E.; Hill, C.J.; Rochford, P.; Fiore, J.; Berlowitz, D.J.; McDonald, C.F. Telerehabilitation for people with chronic obstructive pulmonary disease: Feasibility of a simple, real time model of supervised exercise training. J. Telemed. Telecare 2013, 19, 222-226. [CrossRef]

35. Wardini, R.; Dajczman, E.; Yang, N.; Baltzan, M.; Prefontaine, D.; Stathatos, M.; Marciano, H.; Watson, S.; Wolkove, N. Using a virtual game system to innovate pulmonary rehabilitation: Safety, adherence and enjoyment in severe chronic obstructive pulmonary disease. Can. Respir. J. 2013, 20, 357-361. [CrossRef] [PubMed]

36. Tousignant, M.; Marquis, N.; Page, C.; Imukuze, N.; Metivier, A.; St-Onge, V.; Tremblay, A. In-home Telerehabilitation for Older Persons with Chronic Obstructive Pulmonary Disease: A Pilot Study. Int. J. Telerehabilt. 2012, 4, 7-14. [CrossRef] [PubMed]

37. Gonzalez-Gerez, J.J.; Saavedra-Hernandez, M.; Anarte-Lazo, E.; Bernal-Utrera, C.; Perez-Ale, M.; Rodriguez-Blanco, C. Short-Term Effects of a Respiratory Telerehabilitation Program in Confined COVID-19 Patients in the Acute Phase: A Pilot Study. Int. J. Environ. Res. Public Health 2021, 18, 7511. [CrossRef]

38. Li, J.; Xia, W.; Zhan, C.; Liu, S.; Yin, Z.; Wang, J.; Chong, Y.; Zheng, C.; Fang, X.; Cheng, W.; et al. A telerehabilitation programme in post-discharge COVID-19 patients (TERECO): A randomised controlled trial. Thorax 2021. [CrossRef]

39. Bhatt, S.P.; Patel, S.B.; Anderson, E.M.; Baugh, D.; Givens, T.; Schumann, C.; Sanders, J.G.; Windham, S.T.; Cutter, G.R.; Dransfield, M.T. Video Telehealth Pulmonary Rehabilitation Intervention in Chronic Obstructive Pulmonary Disease Reduces 30-Day Readmissions. Am. J. Respir. Crit. Care Med. 2019, 200, 511-513. [CrossRef] [PubMed]

40. Tsai, L.L.; McNamara, R.J.; Moddel, C.; Alison, J.A.; McKenzie, D.K.; McKeough, Z.J. Home-based telerehabilitation via real-time videoconferencing improves endurance exercise capacity in patients with COPD: The randomized controlled TeleR Study. Respirology 2017, 22, 699-707. [CrossRef]

41. Chan, C.; Yamabayashi, C.; Syed, N.; Kirkham, A.; Camp, P.G. Exercise Telemonitoring and Telerehabilitation Compared with Traditional Cardiac and Pulmonary Rehabilitation: A Systematic Review and Meta-Analysis. Physiother. Can. 2016, 68, $242-251$. [CrossRef] [PubMed]

42. Garvey, C.; Singer, J.P.; Bruun, A.M.; Soong, A.; Rigler, J.; Hays, S. Moving Pulmonary Rehabilitation into the Home: A Clinical Review. J. Cardiopulm. Rehabilt. Prev. 2018, 38, 8-16. [CrossRef] [PubMed]

43. Bierman, R.T.; Kwong, M.W.; Calouro, C. State Occupational and Physical Therapy Telehealth Laws and Regulations: A 50-State Survey. Int. J. Telerehabilt. 2018, 10, 3-54. [CrossRef]

44. Lundell, S.; Holmner, A.; Rehn, B.; Nyberg, A.; Wadell, K. Telehealthcare in COPD: A systematic review and meta-analysis on physical outcomes and dyspnea. Respir. Med. 2015, 109, 11-26. [CrossRef] [PubMed] 